\title{
HIGHER ORDER OPTIMALITY AND DUALITY IN FRACTIONAL VECTOR OPTIMIZATION OVER CONES
}

\author{
SURJEET KAUR SUNEJA, MEETU BHATIA GROVER AND MUSKAN KAPOOR
}

\begin{abstract}
In this paper we give higher order sufficient optimality conditions for a fractional vector optimization problem over cones, using higher order cone-convex functions. A higher order Schaible type dual program is formulated over cones. Weak, strong and converse duality results are established by using the higher order cone convex and other related functions.
\end{abstract}

\section{Introduction}

In the past few years, the field of fractional optimization problems has been of great interest because many optimization problems which arise from practical needs turn out to be of fractional form. These problems have been studied by various researchers. Charnes and Cooper [1] in their classical paper in 1962 introduced the fractional programming problems where the functions concerned are linear in nature. Then Dinkelbach [2] solved nonlinear fractional programming problems using the parametric approach. For linear and concaveconvex fractional programs, a duality theory was developed by Schaible [3]. He proposed a revised version of Dinkelbach's algorithm in [4] using duality theory introduced in [3]. Schaible and Ibaraki [5] gave many economic, noneconomic and indirect applications of fractional programming problems.

Liang et al. [6] presented optimality and duality results for a class of nonlinear fractional programming problems. Later in [7], for the class of multiobjective fractional programming problems, he gave efficiency conditions and duality results using the concept of $(F, \alpha, \rho, d)$ convexity introduced in [6]. Antczak [8] gave a modified objective function method for solving nonlinear multiobjective fractional programming programs and Jayswal et al. [9] established sufficient optimality conditions and duality results.

Received October 8, 2016, accepted January 10, 2017. 2010 Mathematics Subject Classification. 90C32, 90C46.

Key words and phrases. Fractional programming, vector optimization, higher order convex, cones, optimality, duality.

Corresponding author: Muskan Kapoor. 
The study of second and higher order duality is significant due to the computational advantage over the first order duality as it provides tighter bounds for the value of objective function when approximations are used because there are more parameters involved. Mangasarian [10] formulated a class of second and higher order duals for non-linear programs. Higher order duality has been studied by many other researchers like Chen [11], Yang et al. [12], Mishra and Rueda [13], Zhang [14] etc. Higher order cone convex, pseudoconvex, strongly pseudoconvex and quasiconvex functions were introduced by Bhatia [15] and sufficiency and higher order duality results were studied for a vector optimization problem over cones using higher order convex functions. The notions of higher order naturally cone pseudoconvex, strictly cone pseudoconvex and weakly cone quasiconvex functions were introduced by Suneja et al. [16] and the results of Bhatia [15] were extended. Generalized Mond-Weir type higher order dual was formulated and various duality results were established using higher order strongly cone pseudoconvex and higher order cone quasiconvex functions. Higher or$\operatorname{der}(F, \rho, \sigma)$-type I functions were introduced by Suneja et al. [17]. Higher order Mond-Weir and Schaible type duals were formulated for a nondifferentiable multiobjective fractional program and various duality results were established using these functions.

A multiobjective fractional programming problem in which all the concerned functions were taken to be continuously differentiable and the denominator of each objective function consisted of the same scalar function was considered in 2005 by Kim [18] and he gave the necessary and sufficient optimality conditions and saddle point theorems under the generalized invexity assumptions. Then in 2006 he [19] again considered the same problem where the concerned functions were taken to be locally lipschitz. He also introduced the property of generalized invexity for fractional functions and presented necessary and sufficient optimality conditions and duality relations under suitable generalized invexity assumptions.

In this paper a fractional vector optimization problem over arbitrary cones has been considered in which the denominator of each component of the objective function contains the same scalar function and sufficient optimality conditions have been established for a feasible point to be weak minimum, minimum or strong minimum. A higher order Schaible type dual has been formulated and weak, strong and converse duality results have been proved.

\section{Notations and definitions}

Let $K \subseteq R^{m}$ be a closed convex pointed cone with vertex at origin and int $K \neq \varnothing$ where int $K$ denotes the interior of $K$. The positive polar cone $K^{+}$and strict positive polar cone $K^{s+}$ of $K$ are defined as follows:

$$
K^{+}=\left\{y \in R^{m}: x^{T} y \geq 0, \forall x \in K\right\} \text { and } K^{s+}=\left\{y \in R^{m}: x^{T} y>0, \forall x \in K \backslash\{0\}\right\}
$$


Let $g: R^{n} \rightarrow R, f: R^{n} \rightarrow R^{m}$ and $H: R^{n} \times R^{n} \rightarrow R^{m}$ be differentiable functions, where $f=$ $\left(f_{1}, f_{2}, \ldots, f_{m}\right)^{T}$ and $H=\left(H_{1}, H_{2}, \ldots, H_{m}\right)^{T}$. Let $p: R^{n} \times R^{n} \rightarrow R^{n}$ be a vector valued function where $p=\left(p_{1}, p_{2}, \ldots, p_{n}\right)^{T}$.

In this section, we recall some of the basic definitions, which are to be used throughout the paper.

Let $S \subseteq R^{n}$ be an open set, $g: R^{n} \rightarrow R, f: R^{n} \rightarrow R^{m}$, where $f=\left(f_{1}, f_{2}, \ldots, f_{m}\right)^{T}$.

Here, $\nabla f(\bar{x})=\left[\nabla f_{1}(\bar{x}), \nabla f_{2}(\bar{x}), \ldots, \nabla f_{m}(\bar{x})\right]^{T}$ is $m \times n$ Jacobian matrix of $f$ at $\bar{x}$ and for each $i=1,2, \ldots, m, \nabla f_{i}(\bar{x})=\left(\frac{\partial f_{i}(\bar{x})}{\partial x_{1}}, \frac{\partial f_{i}(\bar{x})}{\partial x_{2}}, \ldots, \frac{\partial f_{i}(\bar{x})}{\partial x_{n}}\right)^{T}$ is the $n \times 1$ Gradient vector of $f_{i}$ at $\bar{x}$.

Definition 2.1. The function $g$ is said to be convex at $\bar{x} \in S$ on $S$, if for all $x \in S$,

$$
g(x)-g(\bar{x})-(x-\bar{x})^{T}(\nabla g(\bar{x})) \geq 0
$$

Definition 2.2 ([15]). The function $f$ is said to be higher order $K$-convex at $\bar{x} \in S$ on $S$, with respect to $H$, if for all $x \in S$,

$$
f(x)-f(\bar{x})-(\nabla f(\bar{x}))(x-\bar{x})-\left(\nabla_{p} H(\bar{x}, p)\right)(x-\bar{x})-H(\bar{x}, p)+\left(\nabla_{p} H(\bar{x}, p)\right) p \in K
$$

Definition 2.3 ([15]). The function $f$ is said to be higher order strongly $K$-pseudoconvex at $\bar{x} \in S$ on $S$, with respect to $H$, if for all $x \in S$,

$$
\begin{gathered}
-\left(\nabla f(\bar{x})+\nabla_{p} H(\bar{x}, p)\right)(x-\bar{x}) \notin \operatorname{int} K \\
\Rightarrow \quad f(x)-f(\bar{x})-H(\bar{x}, p)+\left(\nabla_{p} H(\bar{x}, p)\right) p \in K
\end{gathered}
$$

Definition 2.4 ([15]). The function $f$ is said to be higher order $K$-quasiconvex at $\bar{x} \in S$ on $S$, with respect to $H$, if for all $x \in S$,

$$
\begin{aligned}
& f(x)-f(\bar{x})-H(\bar{x}, p)+\left(\nabla_{p} H(\bar{x}, p)\right) p \notin \operatorname{int} K \\
\Rightarrow \quad & -\left(\nabla f(\bar{x})+\nabla_{p} H(\bar{x}, p)\right)(x-\bar{x}) \in K
\end{aligned}
$$

\section{Optimality conditions}

Consider the following fractional vector optimization problem

$$
\begin{aligned}
& \text { (FP) } K \text {-Minimize } \frac{f(x)}{g(x)}=\left[\frac{f_{1}(x)}{g(x)}, \frac{f_{2}(x)}{g(x)}, \ldots, \frac{f_{m}(x)}{g(x)}\right]^{T} \\
& \text { subject to }-h(x)=-\left[h_{1}(x), h_{2}(x), \ldots, h_{p}(x)\right]^{T} \in Q
\end{aligned}
$$

where $f: R^{n} \rightarrow R^{m}, g: R^{n} \rightarrow R$ and $h: R^{n} \rightarrow R^{p}$ are differentiable functions. $K$ and $Q$ are closed convex pointed cones in $R^{m}$ and $R^{p}$ respectively with non-empty interiors. The feasible set of (FP) is given by $X_{0}=\left\{x \in R^{n}:-h(x) \in Q\right\}$. We assume that $g(x)>0$ for every $x \in X_{0}$. 


\section{Remark 3.1.}

(i) If we replace $m$ by $k, p$ by $m$ and we take $K=R_{+}^{k}$ and $Q=R_{+}^{m}$, then our problem (FP) reduces to the problem (MFP) considered by Kim [18] where $X=R^{n}$.

(ii) If we interchange $m$ and $p$ and we take $K=R_{+}^{p}$ and $Q=R_{+}^{m}$, then our problem (FP) reduces to the problem (NMFP) considered by Kim [19] where $X_{0}=R^{n}$.

(iii) If we replace $m$ by $k, p$ by $m$, the function $g$ by $q: R^{n} \rightarrow R$ and if we take $K=R_{+}^{k}$ and $Q=S$, then our problem (FP) reduces to the problem (MFP) considered by Chen et al. [20].

(iv) If we replace $m$ by $k, p$ by $m$ and we take $K=R_{+}^{k}$ and $Q=R_{+}^{m}$, then our problem (FP) reduces to the problem (FP) given by Antczak [8] where $X=R^{n}$.

(v) If we replace $m$ by $k, p$ by $m$ and we take $K=R_{+}^{k}$ and $Q=R_{+}^{m}$, then our problem (FP) reduces to the problem (FP) given by Jayswal et al. [9] where $X=R^{n}$.

Definition 3.1. A point $\bar{x} \in X_{0}$ is called

(i) a weak minimum of (FP), if for every $x \in X_{0}$,

$$
\frac{f(\bar{x})}{g(\bar{x})}-\frac{f(x)}{g(x)} \notin \operatorname{int} K
$$

(ii) a minimum of (FP), if for every $x \in X_{0}$,

$$
\frac{f(\bar{x})}{g(\bar{x})}-\frac{f(x)}{g(x)} \notin K \backslash\{0\}
$$

(iii) a strong minimum of (FP), if for every $x \in X_{0}$,

$$
\frac{f(x)}{g(x)}-\frac{f(\bar{x})}{g(\bar{x})} \in K
$$

(iv) a Benson proper minimumof (FP), if

$$
(-K) \bigcap \overline{\text { cone }}\left(\frac{f\left(X_{0}\right)}{g\left(X_{0}\right)}+K-\frac{f(\bar{x})}{g(\bar{x})}\right)=\{0\}
$$

where for any subset $D$ of $R^{m}, \overline{\text { cone }}(D)$ denotes the closure of cone generated by $D$.

Consider the following vector optimization problem for $v=\left(v_{1}, v_{2}, \ldots, v_{m}\right)^{T} \in R^{m}$.

$(\mathrm{FP})_{v} \quad K$-Minimize $f(x)-g(x) v=\left(f_{1}(x)-g(x) v_{1}, f_{2}(x)-g(x) v_{2} \ldots, f_{m}(x)-g(x) v_{m}\right)^{T}$ subject to $-h(x) \in Q$

Since the constraint in both the problems $(\mathrm{FP})$ and $(\mathrm{FP})_{v}$ is same therefore their feasible sets are also same. Now below we give a lemma which relates the above two problems. 
Lemma 3.1. $\bar{x} \in X_{0}$ is a weak minimum of $(F P)$ if and only if $\bar{x}$ is also a weak minimum of $(\mathrm{FP})_{\bar{v}}$, where $\bar{v}=\frac{f(\bar{x})}{g(\bar{x})}$.

Proof. Suppose $\bar{x}$ is a weak minimum of (FP) and if possible not a weak minimum of $(\mathrm{FP})_{\bar{v}}$, then there exists $x \in X_{0}$ such that $[f(\bar{x})-g(\bar{x}) \bar{\nu}]-[f(x)-g(x) \bar{\nu}] \in$ int $K$ which implies that

$$
\left[f(\bar{x})-g(\bar{x}) \frac{f(\bar{x})}{g(\bar{x})}\right]-\left[f(x)-g(x) \frac{f(\bar{x})}{g(\bar{x})}\right] \in \operatorname{int} K
$$

Since $g(x)>0$, therefore multiplying the above relation by $\frac{1}{g(x)}$, we get

$$
\frac{f(\bar{x})}{g(\bar{x})}-\frac{f(x)}{g(x)} \in \operatorname{int} K
$$

which is a contradiction to the fact that $\bar{x}$ is a weak minimum of (FP). Therefore, $\bar{x}$ is also a weak minimum of $(F P)_{\bar{v}}$.

Conversely, suppose $\bar{x}$ is a weak minimum of $(\mathrm{FP})_{\bar{v}}$ and not a weak minimum of (FP), then there exists $x \in X_{0}$ such that

$$
\frac{f(\bar{x})}{g(\bar{x})}-\frac{f(x)}{g(x)} \in \operatorname{int} K
$$

Since $g(x)>0$ for every $x \in X_{0}$ therefore multiplying the above relation by $g(x)$ and using the fact that $\bar{v}=\frac{f(\bar{x})}{g(\bar{x})}$, we get $[f(\bar{x})-g(\bar{x}) \bar{v}]-[f(x)-g(x) \bar{v}] \in \operatorname{int} K$

which is a contradiction to the fact that $\bar{x}$ is a weak minimum of $(\mathrm{FP})_{\bar{v}}$.

\section{Necessary and sufficient optimality conditions}

Based on the above lemma, we have the following necessary optimality conditions for the problem (FP).

Proceeding on the lines of Lemma 1 given by Suneja et al. [21] we have the following Fritz-John type necessary optimality conditions for a point to be a weak minimum of (FP).

Theorem 3.1. Let $\bar{x} \in X_{0}$ be a weak minimum of (FP). Then there exist $\bar{\lambda} \in K^{+}, \bar{\mu} \in Q^{+}$with $(\bar{\lambda}, \bar{\mu}) \neq(0,0)$ such that

$$
(x-\bar{x})^{T}\left[\nabla\left(\bar{\lambda}^{T} f\right)(\bar{x})-\left(\bar{\lambda}^{T} \bar{v}\right) \nabla g(\bar{x})+\nabla\left(\bar{\mu}^{T} h\right)(\bar{x})\right] \geq 0 \quad \forall x \in R^{n}
$$

and

$$
\left(\bar{\mu}^{T} h\right)(\bar{x})=0
$$


We now establish Kuhn-Tucker type necessary optimality conditions for the problem (FP) by using the slater type cone constraint qualification which is defined as follows.

Definition 3.2. The function $h$ is said to satisfy Slater type cone constraint qualification at $\bar{x} \in R^{n}$, if there exists $\hat{x} \in R^{n}$ such that $-h(\hat{x}) \in \operatorname{int} Q$.

Theorem 3.2. Let $\bar{x} \in X_{0}$ be a weak minimum of (FP). Suppose $h$ satisfies the Slater type cone constraint qualification at $\bar{x}$ and $h$ is $Q$-convex at $\bar{x}$. Then there exist $0 \neq \bar{\lambda} \in K^{+}, \bar{\mu} \in Q^{+}$such that (1) and (2) hold.

Proof. Let $\bar{x}$ be a weak minimum of (FP), then we invoke Theorem 3.1 to deduce that there exist $\bar{\lambda} \in K^{+}, \bar{\mu} \in Q^{+}$with $(\bar{\lambda}, \bar{\mu}) \neq(0,0)$ such that Eq. (1) and Eq. (2) hold.

Since $\bar{\mu} \in Q^{+}$and $h$ satisfies Slater type cone constraint qualification, therefore there exists $\hat{x} \in R^{n}$ such that

$$
\left(\bar{\mu}^{T} h\right)(\hat{x})<0
$$

We have to prove that $\bar{\lambda} \neq 0$.

Let if possible, $\bar{\lambda}=0$, then $\bar{\mu} \neq 0$ and for $x=\hat{x}$, (1) reduces to

$$
(\hat{x}-\bar{x})^{T}\left[\nabla\left(\bar{\mu}^{T} h\right)(\bar{x})\right] \geq 0
$$

Now as $h$ is $Q$-convex at $\bar{x}$, hence

$$
h(\hat{x})-h(\bar{x})-[\nabla h(\bar{x})](\hat{x}-\bar{x}) \in Q
$$

Using the fact that $\bar{\mu} \in Q^{+}$, we get,

$$
\left(\bar{\mu}^{T} h\right)(\hat{x})-\left(\bar{\mu}^{T} h\right)(\bar{x})-(\hat{x}-\bar{x})^{T}\left[\nabla\left(\bar{\mu}^{T} h\right)(\bar{x})\right] \geq 0
$$

Adding Eq. (4) and Eq. (5) and using Eq. (2), we get $\left(\bar{\mu}^{T} h\right)(\hat{x}) \geq 0$ which is a contradiction to Eq. (3).

Hence $\bar{\lambda} \neq 0$.

We now obtain higher order sufficient optimality conditions for (FP) by using higher order cone-convexity and convexity assumptions.

Theorem 3.3. Let $f$ be higher order $K$-convex with respect to $H: R^{n} \times R^{n} \rightarrow R^{m},-g$ be convex, $h$ be higher order $Q$-convex with respect to $G: R^{n} \times R^{n} \rightarrow R^{p}$ at $\bar{x} \in X_{0}$ on $X_{0}$, where $G$ and $H$ are differentiable vector valued functions and $f(\bar{x}) \in K$. Suppose that there exist $0 \neq \bar{\lambda} \in K^{+}$and $\bar{\mu} \in Q^{+}$, such that

$$
(x-\bar{x})^{T}\left[\nabla\left(\bar{\lambda}^{T} f\right)(\bar{x})-\left(\bar{\lambda}^{T} \bar{v}\right) \nabla g(\bar{x})+\nabla_{p}\left(\bar{\lambda}^{T} H\right)(\bar{x}, p)+\nabla\left(\bar{\mu}^{T} h\right)(\bar{x})+\nabla_{p}\left(\bar{\mu}^{T} G\right)(\bar{x}, p)\right] \geq 0
$$




$$
\begin{aligned}
& \left(\bar{\mu}^{T} h\right)(\bar{x})+\left(\bar{\mu}^{T} G\right)(\bar{x}, p)-p^{T} \nabla_{p}\left(\bar{\mu}^{T} G\right)(\bar{x}, p)=0 \\
& \left(\bar{\lambda}^{T} H\right)(\bar{x}, p)-p^{T} \nabla_{p}\left(\bar{\lambda}^{T} H\right)(\bar{x}, p)=0
\end{aligned}
$$

Then $\bar{x}$ is a weak minimum of (FP).

Proof. If $\bar{x}$ is not a weak minimum of (FP) then by Lemma 3.1, $\bar{x}$ is not a weak minimum of $(\mathrm{FP})_{\bar{v}}$ and there exists $x \in X_{0}$ such that $[f(\bar{x})-g(\bar{x}) \bar{v}]-[f(x)-g(x) \bar{v}] \in \operatorname{int} K$.

Since $0 \neq \bar{\lambda} \in K^{+}$, we have

$$
\left(\bar{\lambda}^{T} f\right)(\bar{x})-\left(\bar{\lambda}^{T} \bar{\nu}\right) g(\bar{x})-\left(\bar{\lambda}^{T} f\right)(x)+\left(\bar{\lambda}^{T} \bar{\nu}\right) g(x)>0
$$

As $f$ is higher order $K$-convex at $\bar{x} \in X_{0}$ on $X_{0}$, with respect to $H$, we have

$$
f(x)-f(\bar{x})-\nabla f(\bar{x})(x-\bar{x})-\nabla_{p} H(\bar{x}, p)(x-\bar{x})-H(\bar{x}, p)+\nabla_{p} H(\bar{x}, p) p \in K
$$

Since $\bar{\lambda} \in K^{+}$, we get

$$
\begin{aligned}
& \left(\bar{\lambda}^{T} f\right)(x)-\left(\bar{\lambda}^{T} f\right)(\bar{x})-(x-\bar{x})^{T} \nabla\left(\bar{\lambda}^{T} f\right)(\bar{x})-(x-\bar{x})^{T} \nabla_{p}\left(\bar{\lambda}^{T} H\right)(\bar{x}, p) \\
& -\left(\bar{\lambda}^{T} H\right)(\bar{x}, p)+p^{T} \nabla_{p}\left(\bar{\lambda}^{T} H\right)(\bar{x}, p) \geq 0
\end{aligned}
$$

Using Eq. (8) in the above inequality we have,

$$
\left(\bar{\lambda}^{T} f\right)(x)-\left(\bar{\lambda}^{T} f\right)(\bar{x})-(x-\bar{x})^{T}\left[\nabla\left(\bar{\lambda}^{T} f\right)(\bar{x})+\nabla_{p}\left(\bar{\lambda}^{T} H\right)(\bar{x}, p)\right] \geq 0
$$

Since $h$ is higher order $Q$-convex at $\bar{x} \in X_{0}$ on $X_{0}$, with respect to $G$, we have

$$
h(x)-h(\bar{x})-\nabla h(\bar{x})(x-\bar{x})-\nabla_{p} G(\bar{x}, p)(x-\bar{x})-G(\bar{x}, p)+\nabla_{p} G(\bar{x}, p) p \in Q
$$

Using $\bar{\mu} \in Q^{+}$in above relation and adding Eq. (7), we get

$$
\left(\bar{\mu}^{T} h\right)(x)-(x-\bar{x})^{T}\left[\nabla\left(\bar{\mu}^{T} h\right)(\bar{x})+\nabla_{p}\left(\bar{\mu}^{T} G\right)(\bar{x}, p)\right] \geq 0
$$

Adding Eq. (10) and Eq. (11) and using Eq. (6), we have

$$
\left(\bar{\lambda}^{T} f\right)(x)-\left(\bar{\lambda}^{T} f\right)(\bar{x})-(x-\bar{x})^{T}\left(\bar{\lambda}^{T} \bar{\nu}\right) \nabla g(\bar{x})+\left(\bar{\mu}^{T} h\right)(x) \geq 0
$$

Since $x$ is feasible for (FP), $-h(x) \in Q$, which implies $-\left(\bar{\mu}^{T} h\right)(x) \geq 0$.

Therefore Eq. (12) gives

$$
\left(\bar{\lambda}^{T} f\right)(x)-\left(\bar{\lambda}^{T} f\right)(\bar{x})-(x-\bar{x})^{T}\left(\bar{\lambda}^{T} \bar{v}\right) \nabla g(\bar{x}) \geq 0
$$

Also since $-g$ is convex at $\bar{x} \in X_{0}$ on $X_{0}$, therefore

$$
-g(x)+g(\bar{x})+(x-\bar{x})^{T} \nabla g(\bar{x}) \geq 0
$$


Now, since $f(\bar{x}) \in K$ and $g(\bar{x})>0$ we get

$$
\frac{f(\bar{x})}{g(\bar{x})}\left[-g(x)+g(\bar{x})+(x-\bar{x})^{T} \nabla g(\bar{x})\right] \in K .
$$

Using $\bar{\lambda} \in K^{+}$, we get

$$
-\left(\bar{\lambda}^{T} \bar{v}\right) g(x)+\left(\bar{\lambda}^{T} \bar{v}\right) g(\bar{x})+(x-\bar{x})^{T}\left(\bar{\lambda}^{T} \bar{v}\right) \nabla g(\bar{x}) \geq 0
$$

Adding Eq. (13) and Eq. (14), we get

$$
\left(\bar{\lambda}^{T} f\right)(x)-\left(\bar{\lambda}^{T} f\right)(\bar{x})-\left(\bar{\lambda}^{T} \bar{v}\right) g(x)+\left(\bar{\lambda}^{T} \bar{v}\right) g(\bar{x}) \geq 0
$$

which is a contradiction to Eq. (9).

Hence $\bar{x}$ is a weak minimum of (FP).

Now we give an example to illustrate the above theorem.

Example 3.1. Consider the problem

$$
\text { (FP) } \begin{aligned}
K \text {-Minimize } \frac{f(x)}{g(x)} & =\left[\frac{f_{1}(x)}{g(x)}, \frac{f_{2}(x)}{g(x)}\right]^{T} \\
\text { subject to }-h(x) & =-\left[h_{1}(x), h_{2}(x)\right]^{T} \in Q .
\end{aligned}
$$

where $f: R \rightarrow R^{2}, g: R \rightarrow R$ and $h: R \rightarrow R^{2}$ are defined as follows:

$$
\begin{aligned}
& f(x)=\left(f_{1}, f_{2}\right)^{T}=\left(2 x-e^{-2 x}, e^{-2 x}+e^{-x}+2 x\right)^{T}, K=\left\{(x, y) \in R^{2}: x \leq y \text { and } x \leq 0\right\}, \\
& g(x)=-(x-1)^{4}+6, h(x)=\left(h_{1}, h_{2}\right)=\left(2-7 x^{4}, x^{4}+x+2\right)^{T}, \\
& Q=\left\{(x, y) \in R^{2}: x \leq y \leq-x\right\} .
\end{aligned}
$$

The feasible set of the problem (FP) is $X_{0}=\left[-\frac{1}{2}, 0\right]$.

Let $\bar{x}=0, p=\bar{x}+1, H_{1}(\bar{x}, p)=p(\bar{x}-2), H_{2}(\bar{x}, p)=p(\bar{x}+3), G_{1}(\bar{x}, p)=\bar{x}-p, G_{2}(\bar{x}, p)=$ $\bar{x}^{2}+p$.

Then $f$ is higher order $K$-convex at $\bar{x} \in X_{0}$ on $X_{0}$ with respect to $H$, because for every $x \in X_{0}$, we have

$$
\begin{aligned}
& f(x)-f(\bar{x})-\nabla f(\bar{x})(x-\bar{x})-\nabla_{p} H(\bar{x}, p)(x-\bar{x})-H(\bar{x}, p)+\nabla_{p} H(\bar{x}, p) p \\
& \quad=\left(1-e^{-2 x}, e^{-2 x}+e^{-x}-2\right)^{T} \in K .
\end{aligned}
$$

Also, $-g$ is convex at $\bar{x} \in X_{0}$ on $X_{0}$ because for every $x \in X_{0}$, we have

$$
-g(x)+g(\bar{x})+(x-\bar{x})^{T} \nabla g(\bar{x})=x^{4}-4 x^{3}+6 x^{2} \geq 0
$$

and $g(x)>0$. 
Here, $K^{+}=\left\{(x, y) \in R^{2}: 0 \leq y \leq-x\right\}, Q^{+}=Q$ and $h$ is higher order $Q$-convex at $\bar{x} \in X_{0}$ on $X_{0}$ with respect to $G$, because for every $x \in X_{0}$, we have

$$
\begin{aligned}
& h(x)-h(\bar{x})-\nabla h(\bar{x})(x-\bar{x})-\nabla_{p} G(\bar{x}, p)(x-\bar{x})-G(\bar{x}, p)+\nabla_{p} G(\bar{x}, p) p \\
& \quad=\left(-7 x^{4}+x, x^{4}-x\right)^{T} \in Q .
\end{aligned}
$$

Also there exist $0 \neq \bar{\lambda}=(-10,10)^{T} \in K^{+}$and $\bar{\mu}=(-4,4)^{T} \in Q^{+}$such that for every $x \in X_{0}$, we have

$$
\begin{aligned}
& (x-\bar{x})^{T}\left[\sum_{i=1}^{2} \bar{\lambda}_{i} \nabla f_{i}(\bar{x})-\left(\sum_{i=1}^{2} \bar{\lambda}_{i} \bar{\nu}_{i}\right) \nabla g(\bar{x})+\sum_{i=1}^{2} \bar{\lambda}_{i} \nabla_{p} H_{i}(\bar{x}, p)+\sum_{j=1}^{2} \bar{\mu}_{j} \nabla h_{j}(\bar{x})+\sum_{j=1}^{2} \bar{\mu}_{j} \nabla_{p} G_{j}(\bar{x}, p)\right] \\
& =-12 x \geq 0, \\
& \sum_{j=1}^{2} \bar{\mu}_{j} h_{j}(\bar{x})+\sum_{j=1}^{2} \bar{\mu}_{j} G_{j}(\bar{x}, p)-p^{T}\left(\sum_{j=1}^{2} \bar{\mu}_{j} \nabla_{p} G_{j}(\bar{x}, p)\right)=0, \\
& \sum_{i=1}^{2} \bar{\lambda}_{i} H_{i}(\bar{x}, p)-p^{T}\left(\sum_{i=1}^{2} \bar{\lambda}_{i} \nabla_{p} H_{i}(\bar{x}, p)\right)=0 \\
& \text { and } f(\bar{x})=(-1,2) \in K .
\end{aligned}
$$

Therefore, $\bar{x}$ is a weak minimum of problem (FP).

Theorem 3.4. Let $f$ be higher order $K$-convex with respect to $H: R^{n} \times R^{n} \rightarrow R^{m}$, $-g$ be convex, $h$ be higher order $Q$-convex with respect to $G: R^{n} \times R^{n} \rightarrow R^{p}$ at $\bar{x} \in X_{0}$ on $X_{0}$, where $G$ and $H$ are differentiable vector valued functions and $f(\bar{x}) \in K$. Suppose that there exist $0 \neq \bar{\lambda} \in K^{s+}$ and $\bar{\mu} \in Q^{+}$, such that Eq. (6), Eq. (7) and Eq. (8) hold. Then $\bar{x}$ is a minimum of (FP).

Proof. If $\bar{x}$ is not a minimum of (FP) then $\bar{x}$ is not a minimum of $(\mathrm{FP})_{\bar{v}}$, then there exists $x \in X_{0}$ such that

$$
[f(\bar{x})-g(\bar{x}) \bar{v}]-[f(x)-g(x) \bar{v}] \in K \backslash\{0\} .
$$

Since $\bar{\lambda} \in K^{s+}$ we have,

$$
\left(\bar{\lambda}^{T} f\right)(\bar{x})-\left(\bar{\lambda}^{T} \bar{\nu}\right) g(\bar{x})-\left(\bar{\lambda}^{T} f\right)(x)+\left(\bar{\lambda}^{T} \bar{\nu}\right) g(x)>0
$$

Since $f$ is higher order $K$-convex with respect to $H,-g$ is convex, $h$ is higher order $Q$-convex with respect to $G$ at $\bar{x} \in X_{0}$ on $X_{0}$ and $f(\bar{x}) \in K$, therefore proceeding on the lines of Theorem 3.3 and using $\bar{\lambda} \in K^{s+}$ and $\bar{\mu} \in Q^{+}$, we get

$$
\left(\bar{\lambda}^{T} f\right)(x)-\left(\bar{\lambda}^{T} f\right)(\bar{x})-\left(\bar{\lambda}^{T} \bar{v}\right) g(x)+\left(\bar{\lambda}^{T} \bar{v}\right) g(\bar{x}) \geq 0
$$

which contradicts Eq. (15).

Hence $\bar{x}$ is a minimum of (FP).

Now we give another example to illustrate the above theorem. 
Example 3.2. Consider the problem

$$
\text { (FP) } \begin{aligned}
K \text {-Minimize } \frac{f(x)}{g(x)} & =\left[\frac{f_{1}(x)}{g(x)}, \frac{f_{2}(x)}{g(x)}\right]^{T} \\
\text { subject to }-h(x) & =-\left[h_{1}(x), h_{2}(x)\right]^{T} \in Q .
\end{aligned}
$$

where $f: R \rightarrow R^{2}, g: R \rightarrow R, h: R \rightarrow R^{2}, K, Q, \bar{x}, p, G$ and $H$ are defined the same as in Example 3.1.

Then $f$ is higher order $K$-convex with respect to $H,-g$ is convex, $h$ is higher order $Q$-convex with respect to $G$ at $\bar{x} \in X_{0}$ on $X_{0}$.

Also there exist $0 \neq \bar{\lambda}=(-10,9)^{T} \in K^{s+}$ and $\bar{\mu}=(-1,1)^{T} \in Q^{+}$such that for every $x \in X_{0}$, we have

$$
\begin{aligned}
& (x-\bar{x})^{T}\left[\sum_{i=1}^{2} \bar{\lambda}_{i} \nabla f_{i}(\bar{x})-\nabla g(\bar{x}) \sum_{i=1}^{2} \bar{\lambda}_{i} \bar{\nu}_{i}+\sum_{i=1}^{2} \bar{\lambda}_{i} \nabla_{p} H_{i}(\bar{x}, p)+\sum_{j=1}^{2} \bar{\mu}_{j} \nabla h_{j}(\bar{x})+\sum_{j=1}^{2} \bar{\mu}_{j} \nabla_{p} G_{j}(\bar{x}, p)\right] \\
& \quad=\frac{-107}{5} x \geq 0, \\
& \sum_{j=1}^{2} \bar{\mu}_{j} h_{j}(\bar{x})+\sum_{j=1}^{2} \bar{\mu}_{j} G_{j}(\bar{x}, p)-p\left(\sum_{j=1}^{2} \bar{\mu}_{j} \nabla_{p} G_{j}(\bar{x}, p)\right)=0, \\
& \sum_{i=1}^{2} \bar{\lambda}_{i} H_{i}(\bar{x}, p)-p\left(\sum_{i=1}^{2} \bar{\lambda}_{i} \nabla_{p} H_{i}(\bar{x}, p)\right)=0
\end{aligned}
$$

and $f(\bar{x})=(-1,2)^{T} \in K$

Therefore, $\bar{x}$ is a minimum of problem (FP).

Theorem 3.5. Let $f$ be higher order $K$-convex with respect to $H: R^{n} \times R^{n} \rightarrow R^{m},-g$ be convex, $h$ be higher order $Q$-convex with respect to $G: R^{n} \times R^{n} \rightarrow R^{p}$ at $\bar{x} \in X_{0}$ on $X_{0}$, where $G$ and $H$ differentiable vector valued functions and $f(\bar{x}) \in K$. Suppose that there exist $\bar{\mu} \in Q^{+}$such that Eq. (6), Eq. (7) and Eq. (8) hold with $\bar{\lambda}$ replaced by $\lambda$ for every $\lambda \in K^{s+}$. Then $\bar{x}$ is a strong minimum of $(F P)$.

Proof. Since $f$ is higher order $K$-convex with respect to $H,-g$ is convex, $h$ is higher order $Q$-convex with respect to $G$ at $\bar{x} \in X_{0}$ on $X_{0}$ and $f(\bar{x}) \in K$, there exist $\bar{\mu} \in Q^{+}$such that Eq. (6), Eq. (7) and Eq. (8) hold with $\bar{\lambda}$ replaced by $\lambda$ for every $\lambda \in K^{s+}$, therefore proceeding on the lines of Theorem 3.3, we get

$$
\begin{aligned}
& \left(\lambda^{T} f\right)(x)-\left(\lambda^{T} f\right)(\bar{x})-\left(\lambda^{T} \bar{v}\right) g(x)+\left(\lambda^{T} \bar{v}\right) g(\bar{x})>0 \text { for every } \lambda \in K^{S+}, x \in X_{0} \\
& \lambda^{T}[f(x)-f(\bar{x})-g(x) \bar{v}+g(\bar{x}) \bar{v}] \geq 0 \text { for every } \lambda \in K^{+}, x \in X_{0}
\end{aligned}
$$

This gives

$$
[f(x)-g(x) \bar{\nu}]-[f(\bar{x})-g(\bar{x}) \bar{\nu}] \in\left(K^{+}\right)^{+}=C l K=K \text {, for every } x \in X_{0} .
$$


Hence $\bar{x}$ is a strong minimum of (FP).

We note that every strong minimum of vector optimization problem is a Benson proper minimum of the problem but the converse is not true. Thus, in view of Theorem $3.5, \bar{x}$ is also a Benson proper minimum of (FP).

\section{Higher order Schaible type dual over cones}

We formulate higher order Schaible type dual over cones for our problem (FP) and prove weak, strong and converse duality results for the pair.

$$
\text { (SFD) } \begin{aligned}
& K \text {-Maximize } v=\left(v_{1}, v_{2}, \ldots, v_{m}\right)^{T} \\
& \text { subject to } \\
& \nabla\left(\lambda^{T} f\right)(u)-\left(\lambda^{T} v\right) \nabla g(u)+\nabla\left(\mu^{T} h\right)(u)+\nabla_{p}\left(\lambda^{T} H\right)(u, p)+\nabla_{p}\left(\mu^{T} G\right)(u, p)=0 \\
& \left(\lambda^{T} f\right)(u)-\left(\lambda^{T} v\right) g(u)+\left(\lambda^{T} H\right)(u, p)-p^{T} \nabla_{p}\left(\lambda^{T} H\right)(u, p) \geq 0 \\
& \left(\mu^{T} h\right)(u)+\left(\mu^{T} G\right)(u, p)-p^{T} \nabla_{p}\left(\mu^{T} G\right)(u, p) \geq 0
\end{aligned}
$$

where $v \in K, u \in R^{n}, \lambda \in K^{+} \backslash\{0\}, \mu \in Q^{+}$.

We will give two weak duality results for the dual problem (SFD). In the first one we assume the involved functions to be higher order cone-convex, where as in the other we will use the concepts of higher order strongly cone-pseudoconvexity and higher order conequasiconvexity.

Theorem 4.1 (Weak Duality Theorem). Let $x$ be feasible for (FP) and ( $u, v, \lambda, \mu, p)$ be feasible for (SFD). Suppose that $f$ is higher order $K$-convex with respect to $H,-g$ is convex and $h$ is higher order $Q$-convex with respect to $G$ at $u$, where $H$ and $G$ are differentiable vector valued functions, then $v-\frac{f(x)}{g(x)} \notin \operatorname{int} K$.

Proof. Let, if possible, $v-\frac{f(x)}{g(x)} \in$ int $K$.

Then using $\lambda \in K^{+} \backslash\{0\}$ and $g(x)>0$, we have

$$
\sum_{i=1}^{m} \lambda_{i}\left\{v_{i} g(x)-f_{i}(x)\right\}>0
$$

Since $f$ is higher order $K$-convex at $u$, with respect to $H$ and $\lambda \in K^{+} \backslash\{0\}$, we have

$$
\begin{aligned}
& \sum_{i=1}^{m} \lambda_{i}\left\{f_{i}(x)-f_{i}(u)\right\}-(x-u)^{T}\left\{\sum_{i=1}^{m} \lambda_{i}\left(\nabla f_{i}(u)+\nabla_{p} H_{i}(u, p)\right)\right\} \\
& \quad+p^{T} \sum_{i=1}^{m} \lambda_{i} \nabla_{p} H_{i}(u, p)-\sum_{i=1}^{m} \lambda_{i} H_{i}(u, p) \geq 0
\end{aligned}
$$


Since $h$ is higher order $Q$-convex at $u$, with respect to $G$ and $\mu \in Q^{+}$, we have

$$
\begin{aligned}
& \sum_{j=1}^{p} \mu_{j}\left\{h_{j}(x)-h_{j}(u)\right\}-(x-u)^{T}\left\{\sum_{j=1}^{p} \mu_{j}\left(\nabla h_{j}(u)+\nabla_{p} G_{j}(u, p)\right)\right\} \\
& \quad+p^{T} \sum_{j=1}^{p} \mu_{j} \nabla_{p} G_{j}(u, p)-\sum_{j=1}^{p} \mu_{j} G_{j}(u, p) \geq 0
\end{aligned}
$$

Also since $-g$ is convex at $u, \lambda \in K^{+} \backslash\{0\}$ and $v \in K$, therefore

$$
-\sum_{i=1}^{m} \lambda_{i} v_{i} g(x)+\sum_{i=1}^{m} \lambda_{i} v_{i} g(u)+\left(\sum_{i=1}^{m} \lambda_{i} v_{i}\right)(x-u)^{T} \nabla g(u) \geq 0
$$

Adding Eq. (16)-Eq. (22), we have $\sum_{j=1}^{p} \mu_{j} h_{j}(x)>0$ which is a contradiction to the feasibility of $x$ for $(\mathrm{FP})$.

Hence, $v-\frac{f(x)}{g(x)} \notin \operatorname{int} K$.

Theorem 4.2 (Weak Duality Theorem). Let $x$ be feasible for (FP) and $(u, v, \lambda, \mu, p)$ be feasible for (SFD). Suppose that $f-v g$ is higher order strongly $K$-pseudoconvex with respect to $H$ and $h$ is higher order $Q$-quasiconvex with respect to $G$ at $u$, where $H$ and $G$ are differentiable vector valued functions, then

$$
v-\frac{f(x)}{g(x)} \notin \operatorname{int} K
$$

Proof. Let, if possible, $v-\frac{f(x)}{g(x)} \in \operatorname{int} K$.

Proceeding on the lines of Theorem 4.1, we get

$$
\left(\lambda^{T} v\right) g(x)-\left(\lambda^{T} f\right)(x)>0
$$

Since $x$ is feasible for (FP) and $(u, v, \lambda, \mu, p)$ is feasible for (SFD), therefore

$$
\left(\mu^{T} h\right)(x)-\left(\mu^{T} h\right)(u)-\left(\mu^{T} G\right)(u, p)+p^{T} \nabla_{p}\left(\mu^{T} G\right)(u, p) \leq 0 .
$$

If $\mu \neq 0$, then we have $h(x)-h(u)-G(u, p)+p^{T} \nabla_{p} G(u, p) \notin \operatorname{int} Q$.

Since $h$ is higher order $Q$-quasiconvex at $u$ with respect to $G$, therefore

$$
-(x-u)^{T}\left[\nabla h(u)+\nabla_{p} G(u, p)\right] \in Q
$$

which implies that $-(x-u)^{T}\left[\nabla\left(\mu^{T} h\right)(u)+\nabla_{p}\left(\mu^{T} G\right)(u, p)\right] \geq 0$.

If $\mu=0$, then also the above inequality holds. 
Since $(u, v, \lambda, \mu, p)$ is feasible for (SFD), so from the above inequality we get

$$
-(x-u)^{T}\left[\nabla\left(\lambda^{T} f\right)(u)-\left(\lambda^{T} v\right) \nabla g(u)+\nabla_{p}\left(\lambda^{T} H\right)(u, p)\right] \leq 0 .
$$

which implies that $-(x-u)^{T}\left[\nabla f(u)-v \nabla g(u)+\nabla_{p} H(u, p)\right] \notin \operatorname{int} K$.

Further, $f$ is higher order strongly $K$-pseudoconvex at $u$ with respect to $H$, therefore

$$
f(x)-v g(x)-(f(u)-v g(u))-H(u, p)+p^{T} \nabla_{p} H(u, p) \in K
$$

and hence

$$
\left(\lambda^{T} f\right)(x)-\left(\lambda^{T} v\right) g(x)-\left(\lambda^{T} f\right)(u)+\left(\lambda^{T} v\right) g(u)-\left(\lambda^{T} H\right)(u, p)+p^{T} \nabla_{p}\left(\lambda^{T} H\right)(u, p) \geq 0
$$

Again using the fact that $(u, v, \lambda, \mu, p)$ is feasible for (SFD), we get $\left(\lambda^{T} f\right)(x)-\left(\lambda^{T} v\right) g(x) \geq 0$ which is a contradiction to the Eq. (23).

Hence, $v-\frac{f(x)}{g(x)} \notin \operatorname{int} K$.

Below we prove the strong duality result.

Theorem 4.3 (Strong Duality Theorem). Let $\bar{x} \in X_{0}$ be a weak minimum of (FP). Suppose $h$ satisfies Slater type cone constraint qualification at $\bar{x}, h$ is Q-convex at $\bar{x}$ and $H(\bar{x}, 0)=0=$ $G(\bar{x}, 0), \nabla_{p} H(\bar{x}, 0)=0=\nabla_{p} G(\bar{x}, 0)$ then there exist $0 \neq \bar{\lambda} \in K^{+}, \bar{\mu} \in Q^{+} \operatorname{such}$ that $(\bar{x}, \bar{v}, \bar{\lambda}, \bar{\mu}, \bar{p}=0)$ is feasible for (SFD). Suppose the conditions of Weak Duality Theorem 4.1 or 4.2 are satisfied for each feasible solution $x$ of (FP) and $(u, v, \lambda, \mu, p)$ of (SFD), then $(\bar{x}, \bar{v}, \bar{\lambda}, \bar{\mu}, \bar{p}=0)$ is weak maximum of (SFD).

Proof. Since $\bar{x}$ is a weak minimum of (FP), therefore by Lemma 3.1, $\bar{x}$ is also weak minimum of $(\mathrm{FP})_{\bar{v}}$ where $\bar{v}=\frac{f(\bar{x})}{g(\bar{x})}$. Now therefore by Theorem 3.2, we get $0 \neq \bar{\lambda} \in K^{+}$and $\bar{\mu} \in Q^{+}$such that Eq. (1) and Eq. (2) hold. Choosing $x$ properly, we shall get

$$
\nabla\left(\bar{\lambda}^{T} f\right)(\bar{x})-\left(\bar{\lambda}^{T} \bar{v}\right) \nabla g(\bar{x})+\nabla\left(\bar{\mu}^{T} h\right)(\bar{x})=0 .
$$

Also, $H(\bar{x}, 0)=0=G(\bar{x}, 0), \nabla_{p} H(\bar{x}, 0)=0=\nabla_{p} G(\bar{x}, 0)$.

Thus, $(\bar{x}, \bar{v}, \bar{\lambda}, \bar{\mu}, \bar{p}=0)$ is feasible for (SFD).

Now suppose the conditions of Weak Duality Theorem 4.1 or 4.2 hold for all feasible points of (SFD) and let if possible $(\bar{x}, \bar{v}, \bar{\lambda}, \bar{\mu}, \bar{p}=0)$ be not a weak maximum of (SFD) then there exists a feasible point $(u, v, \lambda, \mu, p)$ of (SFD) such that $v-\bar{v} \in \operatorname{int} K$ or $v-\frac{f(\bar{x})}{g(\bar{x})} \in \operatorname{int} K$ which is a contradiction to the Weak Duality Results. Hence $(\bar{x}, \bar{v}, \bar{\lambda}, \bar{\mu}, \bar{p}=0)$, is weak maximum of (SFD).

Next we prove converse duality result between vector optimization problems (FP) and (SFD). 
Theorem 4.4 (Converse Duality Theorem). Let $(\bar{u}, \bar{v}, \bar{\lambda}, \bar{\mu}, \bar{p})$ be feasible point for (SFD) such that $\bar{u} \in X_{0}$. Suppose that $f$ is higher order $K$-convex with respect to $H$, - gis convex and $h$ is higher order $Q$-convex with respect to $G$ at $\bar{u}$, where $H$ and $G$ are differentiable vector valued functions, then $\bar{u}$ is a weak minimum for (FP).

Proof. Let $\bar{u}$ be not a weak minimum for (FP), then $\bar{u}$ is not a weak minimum of $(\mathrm{FP})_{\bar{v}}$ by Lemma 3.1, where $\bar{v}=\frac{f(\bar{u})}{g(\bar{u})}$, then there exists a feasible solution $\hat{u}$ of $(\mathrm{FP})_{\bar{v}}$ such that,

$$
(f(\bar{u})-\bar{v} g(\bar{u}))-(f(\hat{u})-\bar{v} g(\hat{u})) \in \operatorname{int} \mathrm{K}
$$

Then using $\bar{\lambda} \in K^{+} \backslash\{0\}$, we have

$$
\sum_{i=1}^{m} \bar{\lambda}_{i}\left\{f_{i}(\bar{u})-\bar{v}_{i} g(\bar{u})\right\}+\sum_{i=1}^{m} \bar{\lambda}_{i}\left\{\bar{v}_{i} g(\hat{u})-f_{i}(\hat{u})\right\}>0
$$

Since $f$ is higher order $K$-convex at $\bar{u}$ with respect to $H$, for $x=\hat{u}$ and $\bar{\lambda} \in K^{+}$, we have

$$
\begin{aligned}
& \sum_{i=1}^{m} \bar{\lambda}_{i}\left\{f_{i}(\hat{u})-f_{i}(\bar{u})\right\}-(\hat{u}-\bar{u})^{T}\left\{\sum_{i=1}^{m} \bar{\lambda}_{i}\left(\nabla f_{i}(\bar{u})+\nabla_{p} H_{i}(\bar{u}, \bar{p})\right)\right\} \\
& +\bar{p}^{T} \sum_{i=1}^{m} \bar{\lambda}_{i} \nabla_{p} H_{i}(\bar{u}, \bar{p})-\sum_{i=1}^{m} \bar{\lambda}_{i} H_{i}(\bar{u}, \bar{p}) \geq 0
\end{aligned}
$$

Since $h$ is higher order $Q$-convex at $\bar{u}$ with respect to $G$, for $x=\hat{u}$ and $\bar{\mu} \in Q^{+}$, we have

$$
\begin{aligned}
& \sum_{j=1}^{p} \bar{\mu}_{j}\left\{h_{j}(\hat{u})-h_{j}(\bar{u})\right\}-(\hat{u}-\bar{u})^{T}\left\{\sum_{j=1}^{p} \bar{\mu}_{j}\left(\nabla h_{j}(\bar{u})+\nabla_{p} G_{j}(\bar{u}, \bar{p})\right)\right\} \\
& \quad+\bar{p}^{T} \sum_{j=1}^{p} \bar{\mu}_{j} \nabla_{p} G_{j}(\bar{u}, \bar{p})-\sum_{j=1}^{p} \bar{\mu}_{j} G_{j}(\bar{u}, \bar{p}) \geq 0
\end{aligned}
$$

Also since $-g$ is convex at $\bar{u}, \bar{\lambda} \in K^{+}$and $\bar{v} \in K$, therefore

$$
-\sum_{i=1}^{m} \bar{\lambda}_{i} \bar{v}_{i} g(\hat{u})+\sum_{i=1}^{m} \bar{\lambda}_{i} \bar{v}_{i} g(\bar{u})+\left(\sum_{i=1}^{m} \bar{\lambda}_{i} \bar{v}_{i}\right)(\hat{u}-\bar{u})^{T} \nabla g(\bar{u}) \geq 0
$$

Using feasibility of ( $\bar{u}, \bar{v}, \bar{\lambda}, \bar{\mu}, \bar{p})$ for (SFD) and Eq. (24)-Eq. (27), we have $\sum_{j=1}^{p} \bar{\mu}_{j} h_{j}(\hat{u})>0$ which is a contradiction to the feasibility of $\hat{u}$ for (FP).

Hence, $\bar{u}$ is a weak minimum for (FP).

\section{Acknowledgement}

The authors are grateful to the referee for valuable suggestions in the improvement of the paper. 


\section{References}

[1] A. Charnes and W. W. Cooper, Programming with linear fractional functionals, Nav. Res. Logist. Quart., 9 (1962), 181-186.

[2] W. Dinkelbach, On nonlinear fractional programming, Manag. Sci., 13 (1967), 492-497.

[3] S. Schaible, Fractional programming I, Duality, Manag. Sci., 22 (1976), 858-867.

[4] S. Schaible, Fractional programming II, On Dinkelbach's Algorithm, Manag. Sci., 22 (1976), 868-873.

[5] S. Schaible and T. Ibaraki, Fractional programming, European J. Oper. Res., 12 (1983), 325-338.

[6] Z. A. Liang, H. X. Huang and P. M. Pardalos, Optimality conditions and duality for a class of nonlinear fractional programming problems, J. Optim. Theory Appl., 110 (2001), 611-619.

[7] Z. A. Liang, H. X. Huang and P. M. Pardalos, Efficiency conditions and duality for a class of multiobjective fractional programming problems, J. Global. Optim., 27 (2003), 447-471.

[8] T. Antczak, A modified objective function method for solving multiobjective fractional programming problems, J. Math. Anal. Appl., 322 (2006), 971-989.

[9] A. Jayswal, R. Kumar and D. Kumar, Multiobjective fractional programming problems involving $(p, r)-\rho-(\eta, \theta)-$ invex function, J. Appl. Math. Comput., 39 (2012), 35-51.

[10] O. L. Mangasarian, Second and higher duality in nonlinear programming, Journal of Mathematical Analysis and Applications, 51 (1975), 607-620.

[11] X. Chen, Higher order symmetric duality in non-differentiable multiobjective programming problems, Journal of Mathematical Analysis and Applications, 290 (2004), 423-435.

[12] X. M. Yang, K. L. Teo and X. Q. Yang, Higher order generalized convexity and duality in nondifferentiable multiobjective mathematical programming, Journal of Mathematical Analysis and Applications, 297 (2004), $48-55$.

[13] S. K. Mishra and N. G. Rueda, Higher order generalized invexity and duality in non-differentiable mathematical programming, Journal of Mathematical Analysis and Applications, 272 (2002), 496-506.

[14] J. Zhang, Higher Order Convexity and Duality in Multiobjective Programming, in: A. Aberhard, R. Hill, D. Ralph and B.M. Glover (eds.), Progress in Optimization, Contributions from Australisia, Applied Optimization, 30, Kluwer Academic Publishers, Dordrecht (1989), 101-116.

[15] M. Bhatia, Higher order duality in vector optimization over cones, Optimization Letters, 6 (1) (2012), 17-20.

[16] S. K. Suneja, P. Louhan and M. Bhatia, Higher-order cone-pseudoconvex, quasiconvex and other related functions in vector optimization, Optimization Letters, 7 (4) (2013), 647-664.

[17] S. K. Suneja, M. K. Srivastava and M. Bhatia, Higher order duality in multiobjective fractional programming with support functions, Journal of Mathematical Analysis and Applications, 347 (2008), 8-17.

[18] D. S. Kim, Multiobjective fractional programming with a modified objective function, Commun. Korean Math. Soc., 20 (2005), 837-847.

[19] D. S. Kim, Nonsmooth multiobjective fractional programming with generalized invexity, Taiwan. J. Math., 10 (2008), 467-478.

[20] J. W. Chen, Y. J. Cho, J. K. Kim and J. Li, Multiobjective optimization problems with modified objective functions and cone constraints and applications, J. Glob. Optim., 49 (2011), 137-147.

[21] S. K. Suneja, S. Agarwal and S. Davar, Multiobjective symmetric duality involving cones, Eur. J. Oper. Res., 141 (2002), 471-479.

Department of Mathematics, University of Delhi, Delhi 110007, India.

E-mail: surjeetsuneja@gmail.com

Department of Mathematics, Miranda House, University of Delhi, Delhi 110007, India

E-mail: bhatia.meetu@gmail.com

Department of Applied Mathematics, BPIT, GGSIPU, Delhi 110089, India.

E-mail: muskankapoor22@yahoo.com 